\title{
Auto inflammatory Diseases: The Repercussion of unconstrained Inflammasome activation
}

\author{
Emma M. Creagh" ${ }^{* 1}$, Sinead Kenealy \\ ${ }^{1}$ School of Biochemistry and Immunology, Trinity Biomedical Sciences Institute, Trinity College Dublin, University of \\ Dublin, Dublin, Ireland \\ "Corresponding author: Emma M. Creagh, Ph.D. School of Biochemistry and Immunology, Trinity Biomedical \\ Sciences Institute, Trinity College Dublin, University of Dublin, Dublin, Ireland. Email address: ecreagh@tcd.ie \\ Citation: Emma M. Creagh, Auto inflammatory Diseases: The Repercussion of unconstrained Inflammasome \\ activation published in Med Science Journals.
}

Received Date: July 18, 2021 Accepted Date: Aug 05, 2021 Published Date: Aug 16, 2021

\begin{abstract}
Inflammasome area unit sensors at intervals the innate system that area unit chargeable for the regulation of caspase-1 activation and also the initiation of inflammatory responses following cellular infection or harm. a big variety of chronic inflammatory and metabolic diseases have recently been known to own inflammasome-mediated inflammation as a key driver of their pathogenesis; this space of analysis is beneath intense investigation at the moment. This review focusses on auto inflammatory diseases (AD), a apace increasing cluster of debilitating diseases that area unit related to severe general inflammation. AD ordinarily arise as a results of mutations to genes that code inflammasome elements. inheritable AD area unit comparatively rare as a result of they need totally penetrating mutations; but, they usually gift at birth and last a life. Clinical awareness of AD is lacking and it's believed that, at present, several cases go unknown. This review specifically discusses variety of inflammasome-associated AD and metabolic disorders that give vital insight into our understanding of inflammasome signal pathways. These AD highlight the efficiency of inflammasomes in their ability to initiate and sustain general inflammation. The debilitating symptoms of $\mathrm{AD}$ additionally reveal the intensive consequences of uncontrolled inflammasome activity. Clinical therapies that concentrate on the inflammasome and interleukin-1ß, a product of its activation, within the winning management of $\mathrm{AD}$ and bound metabolic diseases will be mentioned.
\end{abstract}

\section{Introduction}

The system is associate evolutionarily preserved system that has evolved to guard the host from offensive pathogens and cellular harm. whereas the system is crucial in protective the host from a spread of insults, the dysregulation of immune parts is powerfully connected to the event of each autoinflammatory and reaction diseases. Autoinflammatory maladys (AD) are comparatively new class of medicine disease. The clinical term AD was projected in 1999, once solely 2 genes (MEFV and NLRP3) had been genetically related to this malady class. Today, thirty genes are connected to $\mathrm{AD}$, that is that the term still wont to describe this increasing cluster of diseases, caused by the over activation of the innate system. As this is often a comparatively new cluster of diseases, with new clinical subtypes being known on associate current basis, there ar restricted applied math analyses out there on AD. In 2013, a study1 calculable the incidence of AD to be two.83 patients per million folks in Sweden. thanks to their comparatively recent identification and their low incidence rates, it's believed that clinical cases of AD are presently underdiagnosed and hyperbolic clinical awareness of $\mathrm{AD}$ is needed.

Although each autoinflammatory and reaction diseases result from the system offensive the body's own tissues, AD are characterized by intense episodes of inflammation, driven by innate immune cells, and are caused by mutations in genes that regulate immunity. 2 The classical symptoms of auto inflammation are infectious disease attacks, skin rash, and abdominal pain. However, AD symptoms vary greatly across clinical subtypes, and patients will gift with a variety of physical manifestations, as well as mouth ulcers, pathology skin or bone lesions, joint swelling, serositis, and tumor lesions.2 the quantity of sequence mutations related to AD is apace increasing. samples of AD embrace cryopyrin-associated periodic syndrome (CAPS), Blau syndrome, familial Malta fever (FMF), growth mortification issue (TNF) receptor-associated periodic syndrome (TRAPS), and hereditary hyperimmunoglobulinaemia D with periodic fever syndrome (HIDS). The prevalence of a given malady will vary from 1:1,000 folks (Sweet's syndrome) to 1: 1,000,000 (Marshall's syndrome, additionally called periodic fever redness raw throat aphthous [PFAP A] ulcers), and vary between populations. McGonagle and McDermott3 projected a 'continuum model' for medicine diseases in 2006 , group action $\mathrm{AD}$ into a spectrum starting from heritable autoinflammatory disorders to heritable reaction diseases, with inheritable autoinflammatory/autoimmune diseases associated alternative diseases that will have each associate autoinflammatory and a reaction part enclosed at intervals this spectrum. the bulk of mutated genes known thus far connected to heritable AD represent essential innate detector or receptor proteins concerned in inflammatory responses, like NLRP3 for CAPS, NOD2 for Blau, and TNFR1 for TRAPS. each NLRP3 and NOD2 macromolecules belong to the Nod-like Receptor (NLR) protein family, a gaggle of cytosolic detector proteins that are capable of police investigation living thing infection or harm. 


\section{Inflammasomes: Critical Mediators of Inflammation}

Inflammasomes are large complexes of proteins that form to mediate the activation of an inflammatory enzyme, termed caspase-1. Caspase-1 is transcribed as the inactive precursor protein pro-caspase-1, which requires proteolytic processing before the generation of its active form. Once active, caspase- 1 is responsible for the maturation and secretion of interleukin (IL)-1 $\beta$ and IL-18, two potent proinflammatory cytokines that induce fever and interferon (IFN) $\gamma$ secretion, respectively. In addition to the activation of the cytokines IL-1 $\beta$ and IL-18, inflammasome activation also results in a type of cell death, termed pyroptosis. Pyroptosis is an inflammatory form of cell death, mediated by caspase-1-dependent cleavage of an executioner protein, Gasdermin D (GSDMD). Cleaved GSDMD is responsible for forming pores in the cell membrane, mediating the release of proinflammatory cytokines IL-1 $\beta$ and IL-18. Thus, the ultimate outcome of inflammasome activation in cells is acute inflammation, driven by the secretion of potent inflammatory mediators IL-1 $\beta$ and IL-18, and pyroptotic cell death, which also contributes to local tissue inflammation in addition to eliminating damaged and infected cells.

Inflammasomes are giant complexes of proteins that kind to mediate the activation of associate degree inflammatory accelerator, termed caspase-1. Caspase- 1 is transcribed because the inactive precursor macromolecule pro-caspase-1, which needs chemical action process before the generation of its active kind. Once active, caspase-1 is answerable for the maturation and secretion of lymphokine (IL)-1 $\beta$ and IL-18, 2 potent pro-inflammatory cytokines that induce fever and antiviral agent (IFN) $\gamma$ secretion, severally. In addition to the activation of the cytokines IL-1 $\beta$ and IL-18, inflammasome activation conjointly leads to a sort of necrobiosis, termed pyroptosis. Pyroptosis is associate degree inflammatory sort of necrobiosis, mediate by caspase-1-dependent cleavage of associate degree public executioner macromolecule, Gasdermin D (GSDMD). Cleaved GSDMD is answerable for forming pores within the semipermeable membrane, mediating the discharge of pro-inflammatory cytokines IL- $1 \beta$ and IL-18. Thus, the final word outcome of inflammasome activation in cells is acute inflammation, driven by the secretion of potent inflammatory mediators IL-1 $\beta$ and IL-18, and pyroptotic necrobiosis, that conjointly contributes to native tissue inflammation additionally to eliminating broken and infected cells.

Inflammasomes area unit composed of a sensor protein, like bound NLR proteins; associate degree sensor protein, sometimes the ASC protein; and also the protein caspase-1. The sensor proteins are accountable for recognizing animate thing pathogens, like microorganism or viruses, and/or animate thing danger or stress signals, like detection of nuclear factors (e.g., DNA or high motility cluster box one proteins [HMGB1]) within the cytoplasm. Once activated following the popularity of a infectious agent or danger signal, the device supermolecule oligomerises associate degreed triggers the formation of an inflammasome. Inflammasomes usually need a priming step before they will become activated, that is termed Signal one. This priming step is mediate by NFKB signaling, that happens following extracellular infectious agent recognition by a toll-like receptor (TLR) or animate thing recognition by bound styles of NLR, like NOD1/2. Activation of NFאB throughout Signal one ends up in the transcriptional upregulation of inflammasome parts, like NLRP3 and pro-IL-1 $\beta$, the inactive precursor type of IL-1 $\beta$. Signal two involves activation and formation of the inflammasome complicated via matter binding to a device supermolecule (Figure 1). an alternate methodology of NLRP3 inflammasome activation, called the no canonical inflammasome, needs a symbol three, mediate by inflammatory caspases-4/5 in humans (caspase-11 in mice).13 Caspase-4, 5, and eleven area unit accountable for direct recognition of animate thing lipopolysaccharides, which ends up in their future cleavage and activation. In addition to regulation of the no canonical inflammasome, activate caspases-4, 5, and eleven may also initiate pyroptosis, as a result of they're capable of directly process GSDMD.9 many inflammasome complexes are known up to now, as well as those who incorporates NLR device proteins (NLRP1, NLRP3, NLRC4, NLRP6, and NLRP12) and different sensors, like AIM2 and IFI16, that area unit members of the PYHIN supermolecule family.

There are $>23$ distinct NLR genes known within the human order, many of that are concerned within the regulation and activation of inflammasome complexes, that afterward cause the activation and secretion of the unhealthy cytokines IL-1 $\beta$ and IL-18. the foremost wide studied and best characterized of all inflammasomes is that of NLRP3. intensive analysis has elucidated a variety of microorganism and no microbial activators of the NLRP3 inflammasome. NLRP3 has been concerned in sleuthing a excessiveness of microorganism pathogens, together with the respiratory disease a deadly disease, stomatitis virus, microorganism staphylococci aureus, E. coli, plant candida, Aspergillus fumigatus, and parasitic genus Schistosoma mansoni and Dermatophagoides pteronyssinus. in addition, bodily function of particulates, like monosodium salt (MSU) crystals, amyloid- $\beta$, silica, metallic element salt dehydrates, asbestos, and alum have all been reportable to activate NLRP. These nonmicrobial agonists induce metal flow that ends up in consequent NLRP3 activation. Recent studies have reportable NEK7 as a completely unique NLRP3 inflammasome regulator. NEK7, a member of the NIMA-related enzyme family, was originally found to be to blame for control mitotic progression and response to DNA harm however has since been reportable to manage NLRP3 oligomerization, formation of Associate in Nursing ASC speck, and consequent caspase-1 activation downstream of metal flow and reactive O species (ROS). Following their bodily function by innate immune cells, intracellular particulates are thought to break the lysosomal membrane, leading to the discharge of the lysosomal accelerator, Cathepsin B, into the cytoplasm, leading to NLRP3 activation. 


\section{Inflammasome-Mediated Autoinflammatory Diseases}

While inflammasome activation could be a key mechanism to blame for mediating the host innate response following infection and injury, inappropriate inflammasome activity will cause AD. As printed antecedently, a number of the well-characterized AD occur as a results of mutations in inflammasome-associated genes.

Mutations in NLRP3 are coupled to a gaggle of disorders conjointly referred to as CAPS, as well as familial cold autoinflammatory syndrome (FCAS), Muckle-Wells syndrome, and neonatal-onset multisystem inflammatory disorder (also referred to as chronic infantile neurological, cutaneous, and body part syndrome). All 3 CAPS subphenotypes occur as a results of dominantly inheritable gain-of-function mutations within the NLRP3 cistron, that end in general inflammation with blood neutrophilia and fever. Localized neutrophilic inflammation is additionally discovered in varied tissues, like skin, muscles, joints, and liquid body substance. Symptoms common to all or any CAPS patient's area unit rash, periodic fevers, headaches, joint pain, pinkeye, and general unease. FCAS is that the least severe of the CAPS and symptoms, that occur from early infancy, area unit triggered inside a pair of hours once cold exposure and usually subside inside twenty-four hours. FCAS is distinct from cold efflorescence, that is caused by AN allergic response to cold and usually develops later in life. Symptoms in Muckle-Wells syndrome patients, triggered by cold, stress, or different unknown factors, area unit like those of FCAS however can also be among progressive hearing impairment and also the development of malady, thanks to excessive blood serum amyloid production. 34 Neonatal-onset multisystem inflammatory disorder has the best degree of chronic inflammation of all CAPS, with symptoms as well as antiseptic infectious disease, papilloedema, joint issues, hearing impairment, and sometimes mental and physical organic process delays.

Both in vitro and in vivo information support the hypothesis that CAPS-associated NLRP3 mutations end in increased responsiveness of the NLRP3 sensing element macromolecule, resulting in inappropriate inflammasome activation and later secretion of the potent inflammatory mediators, IL-1 $\beta$ and IL-18. Downstream markers of inflammation, like IL-6, \{are also area unit are systematically elevated in patients with FCAS once a light cold even once important will increase in IL-1 $\beta$ and IL-18 are undetectable.39 in addition, pretreatment with anti-IL-1 medical aid will stop the FCAS response to a light cold, suggesting a motive role for IL-1 $\beta$ in mediating the response. Approved and effective treatment choices for CAPS patients currently exist, as interference the action of IL-1 $\beta$ victimization anakinra, rilonacept, or canakinumab area unit effective therapies for all CAPS patients.40 so, the prognosis for all CAPS patients is greatly improved if the AD is diagnosed early and treated with the suitable medical aid before the harm caused by chronic inflammation has any permanent impact on the body. Mutations within the LPIN2 cistron, cryptography the lipin-2 macromolecule, end in another NLRP3-associated AD, termed Majeed syndrome. Lipin-2 has been shown to control each the priming and activation steps of the NLRP3 inflammasome, and Majeed-associated LPIN2 mutations end in end in and increased metal effluence in macrophages, resulting in aberrant NLRP3 activation.

The most common AD is FMF, that happens as a results of mutations within the MEFV cistron, cryptography the pyrin macromolecule. Patients with FMF show longer periods of fever and may have a variety of different symptoms, as well as roseola, arthritis, and serositis.42 because the name suggests, FMF affects populations of Mediterranean descent, significantly Armenian, Turkish, Arabic, and a few Jewish-Israeli populations, during which the carrier rates will be as high as 1:5. The high frequency carrier rates counsel that a selective advantage might exist, and former reports have recommended that the mutated pyrin macromolecule might give enhanced protection against infection, asthma, or allergy.

Inappropriate activation of the pyrin inflammasome is additionally connected to a different unrelated AD: HIDS, conjointly called mevalonate enzyme deficiency. HIDS is caused by mutations within the MVK factor, that encodes for the mevalonate enzyme protein, Associate in Nursing protein to blame for Associate in Nursing early step within the isoprenoid synthesis pathway, catalysing the phosphorylation of mevalonic acid. RhoA signalling relies upon its translocation to the cytomembrane, that is regulated by the isoprenylation of RhoA.56 Defective isoprenoid synthesis happens within the presence of mutations within the mevalonate enzyme protein, leading to loss of RhoA activity. Therefore, the molecular mechanism underlying the inflammatory symptoms of HIDS is additionally planned to be mediate via the pyrin inflammasome.53 In distinction to FMF patients, colchicine is ineffective at preventing HIDS flares, presumably thanks to its inability to activate RhoA, that isn't bound to the membrane thanks to the absence of isoprenylation. Anti-IL-1 $\beta$ therapies area unit the most treatment choice for HIDS patients, though not all patients respond. alternative treatment choices embody anti-inflammatory medicine (NSAID), glucocorticoids, and alternative biologics, like like IL-6 obstruction agents.

\section{Inflammasome Activation in the Pathogenesis of metabolic disease}

The pathological process of the many metabolic disorders, together with arteriosclerosis, kind a pair of DM, obesity, and gout, is powerfully related to chronic inflammation. The inflammasome, and product of inflammasome activation (active IL-1 $\beta$ and IL-18), have recently been known as key mediators of this inflammation, and therefore square measure being intensively studied for his or her ability to modulate the pathological process and progression of metabolic malady. as an example, results from the recent CANTOS trial58 reveal that targeting IL-1 $\beta$-mediated inflammation reduces the danger of adverse internal organ events in patients with a previous history of infarction and high sensitivity CRP level $(>2 \mathrm{mg} / \mathrm{L})$. further analysis from the CANTOS study suggests that inhibition of IL- $1 \beta$ in these patients is additionally related to reduced incidences of carcinoma. this implies that any investigation 
into the utilization the utilization and inflammasome targeting therapies for cancers with a longtime inflammatory part is guaranteed. However, the potential adverse effects that will arise once interference such a potent inflammatory go-between should even be thought of, as patients receiving canakinumab throughout the CANTOS trial had associate degree redoubled incidence of doubtless fatal infections and infection.

The contribution of the NLRP3 inflammasome to metabolic malady has been reviewed in nice detail elsewhere; however, the planned role of the inflammasome within the molecular pathological process of arthritis is summarized here as associate degree example. arthritis could be a chronic disease characterized by deposition of MSU crystals in joints, that type once high concentrations of salt square measure gift. The clinical symptoms of arthritis arise as a results of the inflammatory response that happens following recognition of the MSU crystals. gouty \{arthritis | urarthritis | arthritis\} is believed to be the foremost common reason behind inflammatory arthritis, with associate degree increasing prevalence in each developing and developed countries. Activation of the NLRP3 inflammasome in arthritis has been well investigated and it's believed that TLR activation possibly acts because the 1st priming signal within the response to MSU crystals. Activity of MSU crystals by macrophages, that causes lysosomal injury and sequent activation of the NLRP3 inflammasome constitutes Signal a pair of (Figure 1). IL-1 $\beta$ has been concerned as a key inflammatory go-between to blame for driving the event of arthritis by promoting associate degree inflow of neutrophils into the synovial membrane and joint fluid, that could be a typical hallmark of associate degree inflammatory bout during this malady, medicinal drug therapies like anti-inflammatory and glucocorticoids square measure effective in reducing the symptoms of arthritis. Colchicine is additionally prescribed as a prophylactic treatment or to alleviate ill flares. As delineated antecedently, colchicine inhibits tubule chemical process and, in distinction to the pyrin inflammasome, has been shown to disrupt NLRP3 inflammasome activation. Colchicine additionally inhibits microtubule-based inflammatory cell taxis, secretion of chemokines and cytokines, and activity. several of those cellular processes is found in different diseases involving chronic inflammation, suggesting the potential effectualness of low-dose colchicine in different comorbid conditions related to arthritis, like degenerative arthritis and disorder.

\section{Conclusion}

Anti-IL-1 $\beta$ and inflammasome targeting therapies square measure rising as vital clinical treatments for the management of $\mathrm{AD}$, metabolic diseases, and bound cancers. though inflammasome activation might not be the first cause or major unhealthful issue for several metabolic diseases, recent proof suggests that targeting the inflammatory contribution to those diseases might limit their progression. In distinction, bound inheritable AD, together with CAPS, FMF, and HIDS, are according to arise directly as a results of defective and uncontrolled inflammasome activation. the actual fact that a lot of AD square measure effectively treated by IL-1 $\beta$ blockade and medicines that focus on inflammasome activity highlights the efficiency of inflammasomes in driving chronic inflammation. because the mechanisms governing inflammasome regulation still evolve, therefore too can further targets and therapies to control inflammasome activity throughout malady. However, the importance of controlled, practical inflammation for equilibrium can't be unnoticed. Thus, therapeutic inflammasome inhibition must be balanced against the useful contribution of inflammasomes to natural immunity. 


\section{References}

1. Hemminki K et al. Incidence of hereditary amyloidosis and autoinflammatory diseases in Sweden: Endemic and imported diseases. BMC Med Genet. 2013;14(88):1471-2350

2. Brydges S, Kastner DL. The systemic autoinflammatory diseases: Inborn errors of the innate immune system. Curr Top Microbiol Immunol. 2006;305:127-60.

3. McGonagle D, McDermott MF. A proposed classification of the immunological diseases. PLoS Med. 2006;3(8):1242-8.

4. Shrestha SS, Swerdlow DL, Borse RH, Prabhu VS, Finelli L, et al. (2011) Estimating the burden of 2009 pandemic influenza A (H1N1) in the United States (April 2009-April 2010). Clin Infect Dis 52: S75-S82.

5. Howden LM, Meyer JA (2011) Age and sex composition: 2010. 2010 Census Briefs. Publication C2010BR-03, US Census Bureau.

Submit your next manuscript to Med Science Publishers and benefit from:

$\rightarrow$ Easy online submission process

$\rightarrow$ Rapid peer review process

$\rightarrow$ Online article availability soon after acceptance for Publication

$\rightarrow$ Open access: articles available free online

$\rightarrow$ More accessibility of the articles to the readers/researchers within the field

$\rightarrow$ Better discount on subsequent article submission

Submit your manuscript at

https://medsciencejournals.com/submit-manuscript 